\title{
Contributions de la production d'huile de palme au développement durable
}

\author{
Problématique générale, controverses
}

Hubert OMONT

\author{
TA 179, \\ 4, avenue Agropolis, \\ 34388 Montpellier Cedex 5, \\ France \\ <hubert.omont@cirad.fr>
}

\begin{abstract}
Cultivation of oil palm is the subject of controversy. It is necessary to place these debates in their global context. Three aspects of this context will be particularly developed: the rapid expansion of oil palm cultivation over the last three or four decades, the consequences of this expansion and the controversies and debates it raises, the initiatives to put palm oil in a sustainable development perspective.
\end{abstract}

Key words : palm oil, sustainability, debates, roundtable, deforestation, biodiversity
Au début du xxl ${ }^{\mathrm{e}}$ siècle, la culture du palmier à huile est l'objet de débats : pour ses détracteurs, associer les termes de développement durable et $d$ 'huile de palme sera perçu comme un oxymore, alors que, pour ceux qui la défendent, c'est une évidence pour quelquesuns et un objectif pour beaucoup. Les discussions sont souvent passionnées entre les contradicteurs et il est nécessaire de replacer les débats dans leur contexte global pour mieux comprendre la problématique générale. Trois aspects de ce contexte seront plus particulièrement développés :

- la rapide expansion de la culture du palmier à huile au cours des trois ou quatre dernières décennies ;

- les conséquences de cette expansion et les controverses et débats qu'elle suscite ;

- les initiatives prises par les parties prenantes des débats pour prendre en compte ces polémiques et pour inscrire I'huile de palme dans une perspective de développement durable.

\section{Spectaculaire expansion du palmier à huile}

La conjugaison de l'augmentation de la population et de celle du niveau de vie, surtout dans des pays très peuplés comme la Chine et I'Inde, a provoqué une très forte augmentation de la demande en huile.

La consommation en corps gras par habitant a plus que doublé entre 1975 et 2010, de

$11 \mathrm{~kg}$ par habitant et par an en 1976 à
$24,7 \mathrm{~kg}$ par habitant et par an en 2009 (Oil World ${ }^{1}$ ). Si I'on prolonge cette tendance (+3,5\% par an), elle sera de plus de $50 \mathrm{~kg}$ par habitant et par an en 2030.

Inférieure à 10 millions de tonnes en 1960, la production totale de corps gras a atteint 168,8 millions de tonnes en 2009, dont environ $80 \%$ d'huiles végétales. L'utilisation des huiles se répartit en $74 \%$ pour l'alimentaire, $11 \%$ pour l'énergie, $10 \%$ pour l'oléochimie et $5 \%$ pour d'autres usages (alimentation animale, etc.).

L'huile de palme est celle des huiles végétales qui a le plus progressé, de $11 \%$ du total en 1980 à $34 \%$ en 2009 (figure 1).

À I'horizon 2050, l'augmentation de la demande en huile devrait être comprise entre +34\% (226 millions de tonnes pour une population de 9,15 milliards selon I'ONU aux chiffres de consommation actuels), ou beaucoup plus si on inclut un facteur d'augmentation de niveau de vie et une augmentation simultanée de la consommation par habitant.

\section{Développement récent et rapide du palmier à huile}

L'huile de palme est utilisée traditionnellement depuis très longtemps en Afrique, et son commerce a commencé dès le XIX ${ }^{\mathrm{e}}$ siècle pour satisfaire les besoins de l'industrie en plein essor. Si quelques plantations ont été créées au début

\footnotetext{
${ }^{1}$ Sauf indication contraire, la plupart des chiffres de cet article proviennent des données de Oil World
} Annual 2010 du xx ${ }^{\mathrm{e}}$ siècle au Congo, en Malaisie et en Indonésie, ce n'est qu'à partir des années 1970 que l'expansion a réellement commencé, d'abord en Malaisie puis un peu plus tard en Indonésie. La figure 2 montre l'évolution des surfaces en palmiers adultes entre 1960 et 2009 : la Malaisie est passée de 43000 à plus de 4 millions d'hectares, et l'Indonésie de 70000 à plus de 5,3 millions d'hectares (les surfaces du Nigeria incluent des palmeraies naturelles et subspontanées - wild groves).

Ces deux pays représentent $77 \%$ des surfaces plantées dans le monde, mais la culture du palmier tend à se développer dans d'autres pays d'Asie (Thaïlande, PNG, Philippines), d'Amérique latine (Brésil, Colombie, Équateur, Pérou, Amérique centrale) et d'Afrique (Nigeria, Côte-d'Ivoire, Cameroun, Bénin et, depuis peu, Congo RDC, Libéria et Sierra Leone).

La production a aussi évolué rapidement, de moins de 5 millions de tonnes en 1980 à plus de 45 millions de tonnes en 2009, dont $46 \%$ pour I'Indonésie et $38 \%$ pour la Malaisie (tableau 1).

En Indonésie et en Malaisie, la filière reste dominée par une production de grandes plantations, mais des millions de familles tirent leurs ressources de l'huile de palme, petits planteurs ou employés des grandes sociétés de plantation; les petits planteurs occupent de 35 à $40 \%$ des surfaces plantées en palmier pour $33 \%$ de la production. Ailleurs, ces pourcentages sont variables selon les pays et peuvent monter jusqu'à $90 \%$ (Vermeulen et Goad, 2006).

En 2009, $80 \%$ de la production d'huile de palme ont été commercialisés sur le marché 
Huiles végétales: 39,8 millons de tonnes en 1980

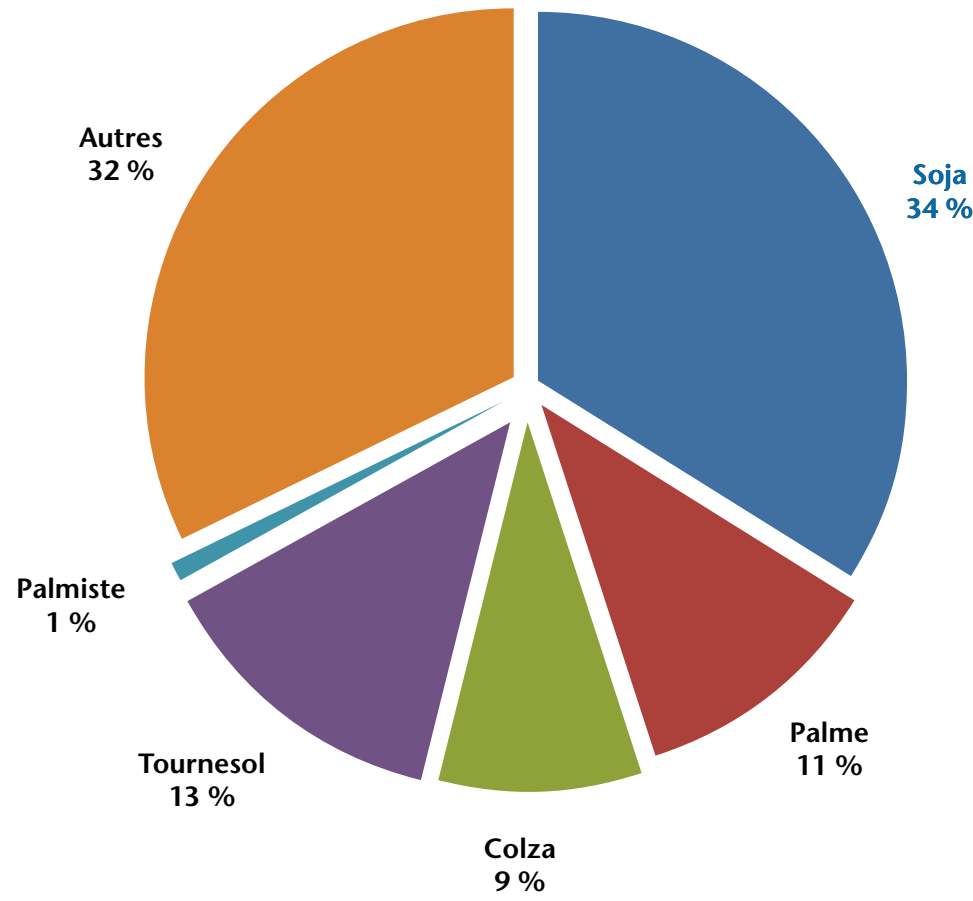

Huiles végétales: 132,8 millons de tonnes en 2009

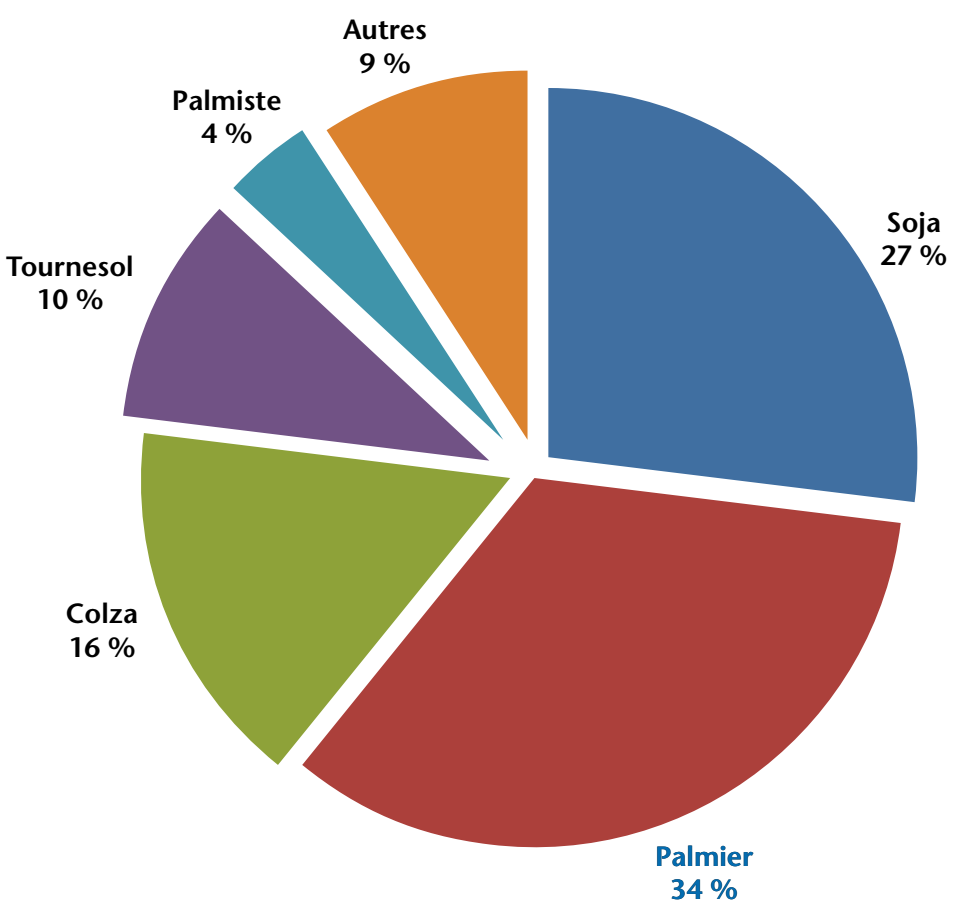

Figure 1. Part relative des différentes huiles végétales (Oil World Annual 2010). mondial (26\% pour le soja); la Malaisie exporte $90 \%$ de sa production, I'Indonésie $76 \%$ :

- exportations : 36,2 millions de tonnes, dont Indonésie $=47 \%$ et Malaisie $=44 \%$;

- importations: Inde $=19 \%$, Chine $=18 \%$, $\mathrm{EU}=16 \%$, États-Unis $=3 \%$.

Les pays du nord consomment moins de $25 \%$ de I'huile de palme produite dans le monde.

\section{Rendement exceptionnel}

Proche de quatre tonnes d'huile par hectare (huile de palme + huile de palmiste), le rendement de la palmeraie est, en moyenne mondiale, dix fois supérieur à celui du soja et quatre fois à celui du colza. Les groupes performants atteignent facilement $6 \mathrm{t} /$ ha sur plusieurs milliers d'hectares, et certaines plantations dépassent même $8 \mathrm{t} / \mathrm{ha}$. Ainsi, pour satisfaire une demande en huile croissante, il faut beaucoup moins de surfaces en palmier qu'en toute autre plante oléagineuse.

\section{Revenus élevés}

L'huile de palme a les coûts de production les moins élevés des huiles végétales, inférieurs de $20 \%$ à ceux du soja et bien inférieurs à ceux du colza (Thoenes, 2006).

Couplé à un prix de I'huile qui, bien qu'inférieur à celui des autres huiles, a sensiblement augmenté ces dernières années (figure 3), cela génère, par hectare, des profits élevés qui attirent beaucoup d'investisseurs, bien au-delà des producteurs traditionnels.

\section{Usages multiples}

L'huile de palme est très polyvalente et peut se substituer à la plupart des autres huiles pour un très grand nombre $d^{\prime}$ usages alimentaires ou industriels selon la répartition suivante :

- environ $80 \%$ pour l'agroalimentaire : huiles de table, huiles de friture, margarines, matières grasses pour boulangerie, pour pâtisserie et pour tout type de préparation alimentaire, etc. ;

- dix-neuf pour cent pour l'oléochimie : cosmétiques, savonneries, lubrifiants et graisses, bougies, produits pharmaceutiques, cuir, surfactants, agrochimie, peintures et laques, électronique, etc. ;

- un pour cent pour le biodiesel, mais cette proportion pourrait augmenter si l'Europe maintient ses directives de substitution en termes de carburants.

\section{Environnement politicoéconomique favorable}

Les gouvernements malais et indonésien ont apporté un fort appui à leurs filières huile de 


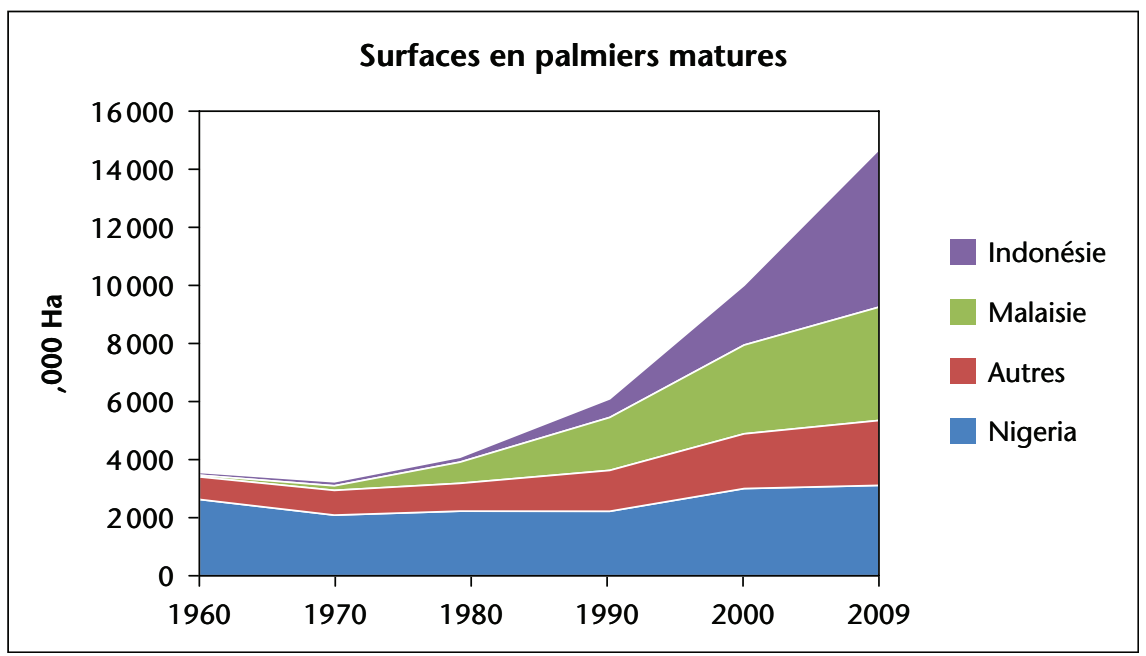

Figure 2. Évolution des surfaces plantées en palmier à huile (FAO STAT).

Tableau 1. Production mondiale d'huile de palme, en million de tonnes.

\begin{tabular}{|llccc|}
\hline Pays & $\mathbf{1 9 8 0}$ & $\mathbf{1 9 9 0}$ & $\mathbf{2 0 0 0}$ & $\mathbf{2 0 1 0}$ \\
\hline Indonésie & 0,7 & 2,4 & 6,9 & 20,9 \\
\hline Malaisie & 2,6 & 6,1 & 10,8 & 17,6 \\
\hline Thailande & 0,01 & 0,2 & 0,5 & 1,3 \\
\hline Nigeria & 0,4 & 0,6 & 0,7 & 0,9 \\
\hline Colombie & 0,07 & 0,2 & 0,5 & 0,8 \\
\hline PNG & 0,04 & 0,1 & 0,3 & 0,5 \\
\hline Équateur & 0,04 & 0,1 & 0,2 & 0,4 \\
\hline Côte-d'Ivoire & 0,2 & 0,3 & 0,3 & 0,3 \\
\hline Autres & 0,8 & 0,8 & 1,7 & 3,2 \\
\hline Total & 4,9 & 10,8 & 21,9 & 45,9 \\
\hline
\end{tabular}

Source : Oil World.

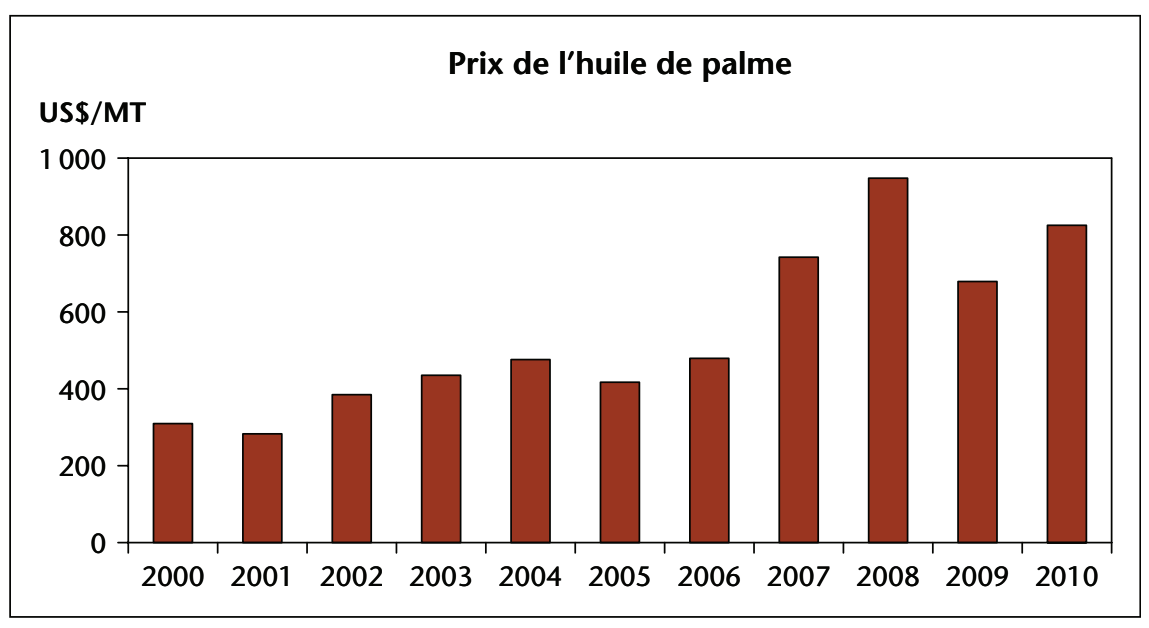

Figure 3. Prix de I'huile de palme (World Bank - Pink sheet, 2010). palme; les protections directes et indirectes ont permis d'attirer les investisseurs locaux et étrangers pour construire une industrie efficiente et dynamique. Recherche et développement, infrastructures générales et maritimes, valeur ajoutée locale, intégration verticale et programmes de marketing et de promotion commerciale ont été les principaux efforts consentis par les États et les filières nationales.

Un haut niveau de concentration du marché (deux pays et un nombre limité de grandes compagnies dans chaque pays) a permis un contrôle permanent du développement et de la modernisation par des acteurs de la filière dynamiques et performants.

Enfin, la filière est peu dépendante du marché de ses sous-produits: I'huile de palmiste se vend sur un marché séparé et la production de tourteaux est peu importante.

Avec autant d'atouts et dans ce contexte de forte demande en huile, l'avenir de l'huile de palme semble donc radieux. II faut cependant composer avec des problèmes liés à la grande concentration du secteur, à une forte dépendance du climat et du marché à l'export, à la stagnation du rendement et à la raréfaction de la main-d'œuvre. Mais, surtout, la filière est confrontée depuis quelques années à de nombreuses questions sur la durabilité de ce développement dans ses aspects sociaux et environnementaux.

\section{Controverses autour de I'huile de palme}

L'expansion rapide des zones cultivées, surtout en Indonésie, n'a commencé à attirer l'attention des grandes ONG environnementales qu'en 1997, au moment « où le monde a pris feu »(WWF, 1997), lorsque de gigantesques incendies ont gravement perturbé le Sud-Est asiatique. L'utilisation du feu pour préparer les terrains avant $d^{\prime} y$ planter des palmiers à huile a été identifiée comme l'une des principales causes de ces incendies en Indonésie.

Plusieurs études de WWF, de UICN, de Greenpeace, de Friends of the Earth ont ensuite montré l'influence de l'expansion du palmier à huile sur la déforestation et la perte de biodiversité qui en découle, mais aussi sur les conflits fonciers et sociaux qui l'accompagnent. Ces études ont aussi accusé certains acteurs de la filière, grands groupes industriels et banques en particulier, de contribuer sans discernement au développement des plantations et à leurs effets négatifs.

Des campagnes contre le développement de I'huile de palme ont alors commencé à apparaître, d'abord sur Internet, puis dans la presse et dans les médias. Et de nouveaux éléments 
sont apparus dans les débats : le changement climatique, la compétition alimentationénergie. L'impact des acides gras saturés de I'huile de palme sur la santé, déjà utilisé par ses détracteurs à la fin $\mathrm{du} x \mathrm{x}^{\mathrm{e}}$ siècle, a de nouveau été mis en avant par les médias lors de ces campagnes.

\section{Huile de palme et déforestation}

Les plantations de palmiers sont sans conteste responsables ces dernières années de la déforestation de grandes étendues de forêts primaires ou secondaires, avec des conséquences négatives de la conversion d'habitat, en particulier sur la perte de biodiversité ; ce phénomène est surtout polémique en Indonésie et se développe pour plusieurs raisons :

- la vente du bois récupéré sur la concession est lucrative dans un marché porteur, et paye tout ou partie de l'installation des palmiers;

- les sols, après défriche de la forêt, sont plus fertiles que les zones préalablement déforestées, souvent envahies par des adventices de type Imperata cylindrica qu'il faut éradiquer ;

- il n'y a pas d'incitations à planter préférentiellement sur des zones déjà déforestées, ni d'obligations réglementaires pour contraindre les compagnies à le faire, avec des conditions $d^{\prime}$ appropriation du foncier souvent plus compliquées ;

- il y a, en revanche, obligation de mettre rapidement en valeur les concessions attribuées sous peine d'en perdre la jouissance.

Certaines compagnies prennent le prétexte de projets de plantation de palmier pour se faire attribuer des concessions, coupent le bois et disparaissent sans planter le moindre palmier.

Ces déforestations ont un impact encore plus violent sur les tourbières, écosystèmes particulièrement fragiles et formidable réserve de carbone. Le gouvernement indonésien vient de décréter un moratoire pour suspendre les plantations sur tourbes (10/2010 http://www. Igslaw.co.id).

Mais le palmier n'est pas responsable de toutes les surfaces déforestées: en Indonésie, où la situation est la plus préoccupante, les ONG estiment que la déforestation concerne 2,5 millions d'hectares chaque année ; le palmier a été planté à un rythme de 0,2 million d'hectares par an de 1995 à 2005 et de 0,4 million d'hectares ces dernières années, soit $16 \%$ seulement du total de la surface défrichée.

La surface totale plantée en palmiers dans le monde n'est que de 15 millions d'hectares, contre plus de 90 millions pour le soja et environ 50 millions pour le colza. Henson et Chang (2003) estiment que le palmier ne contribue que pour moins de $3 \%$ du total des déforestations sur la planète.

\section{Huile de palme et biodiversité}

Les préoccupations en termes de biodiversité sont directement liées au problème de la disparition de la forêt, car certaines zones d'expansion du palmier, en Asie du Sud-Est surtout, contiennent de grandes concentrations d'écosystèmes et d'espèces rares ou en danger (" hotspots » Sundaland et Îles Wallaces).

Jusqu'à récemment, les défrichements se faisaient sans discernement et il n'y avait pas $\mathrm{d}^{\prime}$ attention particulière portée à la protection des zones à haute valeur de conservation $\left(\mathrm{FSC}^{2}\right)$.

En outre, la plantation de grandes étendues de palmier a de lourdes conséquences sur la structure de I'habitat, en particulier pour certaines espèces qui ont besoin de beaucoup d'espace : éléphant, tigres, orang-outang pour citer les espèces les plus emblématiques (Koh et Wilcove, 2008). Au-delà des conflits accrus entre l'homme et les animaux, les risques d'extinction sont sérieux pour certaines espèces du fait de la disparition ou de la fragmentation de leur habitat (Fitzherbert et al., 2008).

\section{Huile de palme et conflits sociaux}

De nombreux conflits sont apparus avec des communautés locales qui se considèrent spoliées par les compagnies qui obtiennent des concessions de la part des autorités sans que la question de leurs droits coutumiers ait été éclaircie (Indonésie, Malaisie, Colombie); droits fonciers inexistants ou ambigus, modalités d'acquisition des terres et utilisation des terres (valeur sacrée par exemple) sont les principaux sujets de discorde (Colchester et al., 2000, 2006).

La survie des populations rurales les plus pauvres, qui dépendent souvent des ressources de la forêt pour leur subsistance, peut être mise en péril par la déforestation pour l'implantation de palmeraies ; les incohérences des lois foncières et l'absence de dialogue sont les principales sources de conflits (Rautner et al., 2005).

Les rapports de force sont souvent déséquilibrés entre les planteurs villageois et les usines qui traitent leurs productions, principalement dans les pays où la filière a été libéralisée, perdant ainsi I'arbitrage des États (Aleksander, 2009 ; Rahman et al., 2008).

Enfin, et même si des progrès notables ont été constatés ces dernières années, les lois sociales ne sont pas toujours appliquées dans certaines plantations industrielles.

\footnotetext{
${ }^{2}$ Forest Stewardship Council.
}

Huile de palme

\section{et changement climatique}

Le palmier peut avoir des effets négatifs ou positifs sur le changement climatique selon la façon dont il est conduit :

- effet négatif lorsqu'il y a utilisation du feu pour la préparation du terrain, ce qui peut aussi provoquer des pollutions atmosphériques graves (1997 en Asie) : cette pratique particulièrement néfaste sur tourbes est aujourd'hui interdite à peu près partout ;

- effet négatif de la libération de méthane par les bassins de décantation des effluents si rien n'est fait pour le capter ;

- effet positif par stockage de carbone si les palmiers sont plantés sur des zones sans forêts, savanes ou surfaces déjà déforestées ;

- effet positif par économie d'énergie fossile si le méthane produit par le traitement des effluents est capté et permet de générer de l'énergie.

\section{Compétition alimentation énergie}

La tension sur le marché des huiles est pour partie liée à l'émergence des biocarburants, directement par la transformation de corps gras en biodiesel, ou indirectement par la conversion de surfaces en cultures oléagineuses vers d'autres spéculations destinées à produire des biocarburants; en 2009, le biodiesel représente plus de $10 \%$ du total de la consommation des huiles, et plus de $50 \%$ de l'augmentation de la demande en 2009/2010 (Oil World Annual, 2010).

Mais on ne peut pas encore vraiment parler de réelle compétition, car la part d'huile de palme destinée à la production de biodiesel ne représente qu'environ $1 \%$ seulement de la production (84\% du biodiesel issu des huiles végétales provient du colza, $13 \%$ du tournesol et $3 \%$ des autres huiles - Thoenes, 2006).

Bien que probablement la plus compétitive, I'huile de palme est pénalisée par les subventions accordées aux autres oléagineux et par certains aspects techniques liés à son point de fusion plus élevé.

La Malaisie a largement investi ces dernières années dans des unités de production de biodiesel, en visant principalement le marché européen; mais l'augmentation du prix de I'huile et les exigences environnementales de certains acheteurs remettent en question la rentabilité de ces investissements et de nombreuses unités tournent très en dessous de leurs capacités.

En outre, les questionnements sur les niveaux de taxes qui pourront leur être appliqués par certains pays et les interrogations globales sur le bien-fondé des biocarburants contribuent à 
rendre les perspectives incertaines pour le moment.

\section{Huile de palme et santé}

L'huile de palme est souvent décriée pour sa forte teneur en acides gras saturés, particulièrement en acide palmitique, molécule qui s'accumule lorsque I'alimentation est déséquilibrée. Mais les relations entre acides gras et obésité ou maladies cardiovasculaires sont complexes, et les résultats publiés sont souvent contradictoires et très difficilement transférables d'une espèce animale à l'autre. Comme souvent, " c'est la dose qui fait le poison ».

En outre, et même si ces résultats demandent à être confirmés chez l'homme adulte, dans I'huile de palme comme dans le beurre de cacao, les acides gras saturés ne se trouveraient pas en position digestible sur le glycérol. Enfin, I'huile de palme étant naturellement hydrogénée, l'industrie apprécie l'absence d'acides gras «trans ", les plus nocifs, ce qui explique que les importations d'huile de palme aux États-Unis aient plus que triplé depuis l'obligation d'étiquetage de leur présence dans les produits alimentaires.

\section{Vers une production durable d'huile de palme}

Pour faire face à ces défis majeurs, des professionnels de la filière et des ONG se sont rassemblés au sein de l'initiative RSPO (Roundtable for Sustainable Palm Oil), créée en 2004, pour travailler à mettre en œuvre une politique de production, d'échanges et de consommation de I'huile de palme qui s'inscrive dans une perspective de développement durable (Omont, 2005, 2006).

C'est une initiative multiacteurs qui a commencé avec 6 membres fondateurs et comprend 485 membres en septembre 2010. Les membres ordinaires sont répartis en sept catégories : producteurs, transformateurs et négociants, industrie, banques, détaillants, ONG/environnementnature, ONG/social ; il y a aussi des membres associés (notamment la recherche). RSPO reçoit constamment des demandes d'adhésion dans toutes les catégories.

RSPO a adopté, en 2005, 8 principes et 39 critères pour définir la notion d'huile de palme durable et répondre à la plupart des polémiques qui font débat; par exemple :

- déforestation: pas de développement de nouvelles plantations sur forêt primaire ou dans des zones à haute valeur de conservation ; moratoire sur les plantations sur tourbes, etc. ; - impacts sociaux : pas de préjudice de la plantation pour les populations locales; respects des droits des plantations familiales et des travailleurs de plantations ;

- changement climatique : interdiction des feux pour la préparation du terrain ; traitement des effluents sans rejets de gaz à effet de serre, etc. La mise en œuvre de ces principes et critères est précisée par des indicateurs génériques qui sont progressivement adaptés aux conditions locales par des interprétations nationales; des indicateurs spécialement adaptés au cas particulier des plantations familiales sont également discutés. Un code de conduite a été accepté par tous les membres.

Un système de certification a été adopté et des mécanismes de commercialisation adaptés ont permis la mise sur le marché des premiers litres d'huile certifiée $\left(\mathrm{CSPO}^{3}\right)$ en novembre 2007 (Cheng Hai, 2010). En novembre 2010 (www.rspo.eu/market/index.html), plus de 3,2 millions de tonnes de CSPO ont été produites, mais seulement 1,4 million de tonnes ont trouvé acheteur. Vingt et une sociétés (75 unités de production) ont été certifiées pour la gestion durable de leurs plantations, et 56 entreprises de la filière ont reçu une certification.

Cette initiative, qui concerne aujourd'hui $40 \%$ de la production, a créé une forte dynamique de groupe dans la filière, mais elle n'a pas éteint toutes les polémiques. Certaines ONG relèvent des contradictions dans le comportement de plusieurs compagnies (certifiées pour une part de leurs activités, et en dehors des critères pour $d^{\prime}$ autres) et regrettent que RSPO n'aille pas assez loin sur certains aspects de la durabilité. À l'inverse, certains producteurs considèrent qu'il s'agit d'entraves au libre commerce, (Pushparajah, 2010).

Dans une récente revue de la performance de RSPO, Laurance et al. (2010) ont identifié plusieurs faiblesses, tels la structure de gouvernance trop dominée par l'industrie, un code de conduite peu performant, une capacité organisationnelle inadéquate et l'incapacité à promouvoir une interdiction générale de la déforestation. Mais cette initiative reste pour le moment la meilleure voie pour améliorer la durabilité de la filière s'il est possible de corriger tout ou partie de ces faiblesses.

\section{Conclusion}

Malgré la création de RSPO et les succès notables qu'on peut attribuer à cette table ronde, notamment en termes de dialogue entre les parties, la situation actuelle reste conflictuelle et le débat reste parfois vif entre les contradicteurs. Mais l'ampleur de la demande semble

${ }^{3}$ Certified Sustainable Palm Oil bien rendre inéluctable le développement du palmier à huile dans les prochaines années.

Dans leur analyse des accusations de greenwashing pour les défenseurs prodéveloppement et de blackwashing pour les écologistes, Koh et al. (2010), montrent que les uns et les autres usent d'arguments pas toujours irréfutables qui, finalement, peuvent brouiller leur image et ne servent pas forcément l'objectif de durabilité.

Finalement, et au-delà des polémiques, I'huile de palme produite selon les principes et critères proposés par RSPO peut et doit contribuer à faire face à une partie de la forte demande en corps gras prévue pour les décennies à venir. Elle peut aussi concourir à réduire la pauvreté de façon significative, surtout si l'accent est mis sur le développement des petites plantations (Rist et al., 2010). La vigilance reste cependant de mise, notamment pour certains aspects sociaux ou environnementaux qui continuent à poser problème pour certains acteurs de la filière.

\section{RÉFÉRENCES}

Aleksander C. The future of nucleus-plasma partnership. Presentation at the RSPO Task Force for Smallholders meeting, $1^{\text {st }}$ November, 2009, Kuala Lumpur.

Cheng Hai T. Key sustainability issues in the palm oil sector. A discussion paper for multistakeholders consultation. World Bank, 2010.

Colchester M, Wee AP, Wong MC, Jalong T. Land is life: land rights and oil palm development in Sarawak. Forest Peoples Programme \& SawitWatch, 2000.

Colchester M, Jiwan N, Andiko Sirait M, Firdaus AY, Surambo A, Pane H. Promised land: palm oil and land acquisition in Indonesian - Implications for local communities and indigenous peoples. Forest Peoples Programme \& SawitWatch, 2006.

Fitzherbert EB, Struebig M], Morel A, et al. How will oil palm expansion affect biodiversity? Trends Ecol Evol 2008 ; 23 : 538-45.

Greenpeace. How the palm oil industry is cooking the climate. Greenpeace International, The Netherlands, 2007.

Henson IE, Chang KC. Oil palm plantations and forest loss: an objective appraisal. In: Proceedings of the PIPOC 2003 International PalmOilCongress, Malaysian Palm Oil Board, 2003 : 960-74.

Koh LP, Wilcove DS. Is oil palm agriculture really destroying tropical biodiversity? Conservation Letters $2008 ; 1: 60-4$.

Koh LP, Ghazoul J, Butker RA, et al. Wash and spin cycle threats to tropical biodiversity. Biotropica $2010 ; 42: 67-71$.

Laurance WF, Koh LP, Butler R, et al. Improving the performance of the Roundtable on Sustainable Palm Oil for nature conservation. Conservation Biology. Society for Conservation Biology, 2010. 
Oil World Annual, 2010.

Omont H. Roundtable on Sustainable Palm Oil rSPO. The second RSPO meeting in Jakarta in October 2004 . OCL $2005 ; 12$ : 125-8.

Omont H. La troisième table ronde de RSPO-RT3, à Singapour en novembre 2005. OCL 2006; 13: 229-32.

Pushparajah E. Environmental concerns or trade barriers? The Planter $2010 ; 86$ : 77-8 (Guest editorial).
Rahman A, Abdullah R, Shariff FM, Simeh MA. The Malaysian palm oil supply chain: the role of the independent smallholder. Oil Palm Industry Economic Journal $2008 ; 8: 17-27$.

Rautner M, Hardiono M, Alfred RJ. Borneo: treasure island at risk - Status of forest, wildlife and related threats on the island of Borneo. WWF Germany, 2005.

Rist L, Feintrenie L, Levang P. The livelihood impacts of oil palm: smallholders in Indonesia. Biodivers Conserv $2010 ; 19: 1009-24$.
RSPO: www.rspo.org - www.rspo.eu

Thoenes P. Biofuels and commodity markets - palm oil focus. FAO, Commodities and Trade Division, 2006.

Vermeulen S, Goad N. Towards better practice in smallholder palm oil production. Natural resource issues series $n^{\circ} 5$. London, UK: International Institute for Environment and Development, 2006.

WWF International. The year the world caught fire. Discussion Paper. WWF International, 1997. 